\title{
Surface Reconstruction in Gradient-Field Domain Using Compressed Sensing
}

\author{
Mohammad Rostami, Student Member, IEEE, Oleg V. Michailovich, Member, IEEE, \\ and Zhou Wang, Fellow, IEEE
}

\begin{abstract}
Surface reconstruction from measurements of spatial gradient is an important computer vision problem with applications in photometric stereo and shape-from-shading. In the case of morphologically complex surfaces observed in the presence of shadowing and transparency artifacts, a relatively large dense gradient measurements may be required for accurate surface reconstruction. Consequently, due to hardware limitations of image acquisition devices, situations are possible in which the available sampling density might not be sufficiently high to allow for recovery of essential surface details. In this paper, the above problem is resolved by means of derivative compressed sensing (DCS). DCS can be viewed as a modification of the classical CS, which is particularly suited for reconstructions involving image/surface gradients. In DCS, a standard CS setting is augmented through incorporation of additional constraints arising from some intrinsic properties of potential vector fields. We demonstrate that using DCS results in reduction in the number of measurements as compared with the standard (dense) sampling, while producing estimates of higher accuracy and smaller variability as compared with CS-based estimates. The results of this study are further supported by a series of numerical experiments.
\end{abstract}

Index Terms-Photometric stereo, shape-from-shading, 3D surface reconstruction, derivative compressed sensing, Poisson equation.

\section{INTRODUCTION}

$\mathbf{R}$ ECONSTRUCTION of a 3D surface from its gradient field is an important problem in computer vision with areas of applications including photometric stereo (PS) [2], [3], shape-from-shading (SFS) [4], and surface inspection [5]. In these applications, a 3D surface of interest $(x, y, z(x, y))$, is recovered from the measurements of the spatial gradient of a (surface) depth function $z(x, y)$. Existing algorithms generally follow a two stage procedure [6]. First, the gradient field $\left(z_{x}, z_{y}\right)$ is measured/calculated at each point $(x, y)$ and then the depth information $z(x, y)$ is obtained through

Manuscript received June 7, 2014; revised December 1, 2014 and March 1, 2015; accepted March 1, 2015. Date of publication March 6, 2015; date of current version March 23, 2015. This work was supported by the Natural Sciences and Engineering Research Council of Canada. The associate editor coordinating the review of this manuscript and approving it for publication was Dr. Anthony Vetro.

M. Rostami is with the Department of Electrical and Systems Engineering, University of Pennsylvania, Philadelphia, PA 19104 USA (e-mail: m2rostam@uwaterloo.ca).

O. V. Michailovich and Z. Wang are with the Department of Electrical and Computer Engineering, University of Waterloo, Waterloo, ON N2L 3G1, Canada (e-mail: olegm@uwaterloo.ca; z.wang@ece.uwaterloo.ca).

Color versions of one or more of the figures in this paper are available online at http://ieeexplore.ieee.org.

Digital Object Identifier 10.1109/TIP.2015.2409565 integration of the gradient field. In particular, under some reasonable assumptions on the light source and object reflection properties, the unit normal to such a surface can be calculated from its grey-scale representation [7]. Subsequently, the normal can be used to recover its corresponding partial derivatives, followed by reconstructing an approximation of the original surface through the solution of a Poisson equation using either calculus of variation [8], direct integration [9], or frequency-domain algorithms [10].

A practical difficulty in implementation of the abovementioned techniques stems from the necessity to deal with relatively large sets of gradient data which can be computationally expensive. Typically, such dense data sets are required to allow for accurate reconstruction of fine surface details, which are often occluded due to shadowing and transparency artifacts [11], [12]. In such cases, improving the acquisition requirements of the hardware in use through reducing the sampling density would unavoidably produce aliasing artifacts, while improving hardware sampling rate might not be viable. Fortunately, advances in computational harmonic analysis offer a means to overcome the above limitation, while allowing for accurately recovering digital signals from their subcritical measurements. This method - known as compressed sensing (CS) [13], [14] - has already revolutionized vast areas of applied sciences, and computer vision in particular [15]. In the current manuscript we propose to solve the problem of surface reconstruction within the framework of CS so as to improve reconstruction quality without increasing the sampling rate. The resulting estimates of the gradient field can be subsequently used to reconstruct the original 3D surface.

It should be noted that the original CS formulation does not incorporate arbitrary a priori information on the interrogated signals, apart from requiring them to be sparsely representable in a predefined basis. In particular, at the case at hand, it seems natural to reconstruct the surface gradient using the fact that the latter constitutes a potential vector field. When subjected to such side information, the classical CS setup transforms into its specific instance - known as derivative compressed sensing (DCS) [16] - which is in the heart of the present study. In this paper, we use DCS as a tool for 3D surface recovery. We improve the accuracy of 3D surface recovery and provide some justification why DCS outperforms classic CS. We then use an efficient method for reconstruction of 3D surfaces from the sub-critical (incomplete) measurements of their spatial gradients [17]. In addition to detailing a computationally efficient algorithm for practical implementation of DCS [17], 
it is shown how the latter can be used to improve the reconstruction quality of the standard CS [13], [14], while resulting in substantial reduction in sampling density. Furthermore, our results indicate that DCS can be used used to improve resolution of surface recovery data acquisition devices.

The rest of the paper is organized as follows. Section II summarizes some basic technical preliminaries on compressed sensing. In Sections III and IV, we explain the proposed scheme and describe a reconstruction algorithm for practical solution of the problem. Experimental results for both synthetic and real world data are presented in Section V, while Section VI finalizes the paper with a discussion on algorithm limitations and conclusions.

\section{Classic Compressive Sensing}

Compressed sensing (CS) is a technique used for reconstruction of signals-of-interest from their sub-critical (incomplete) measurements [13], [14]. This technique has been been applied to improve many challenging problems in image processing [18]-[20] and computer vision [12], [21]-[23]. CS can be used to improve data acquisition in imaging devices. Surface meteorology is a particular case of surface reconstruction which deals with measuring small-scale features on surfaces and thus a dense sampling rate is required. CS has been applied to improve data acquisition in this application [12]. In addition, CS not only can improve data acquisition, but also can be used to incorporate sparsity as side information about the source signal. In [23], authors have used sparsity as prior information to improve robustness of surface recovery against outliers and noise. These potential benefits motivates us to apply DCS for surface recovery.

At a fundamental level, CS exploits sparsity as a prior on the signal to be estimated and recovers an approximation to the signal as a unique solution to a convex optimization problem. Let $\mathbf{x} \in \mathbb{R}^{n}$ be a source signal which has a sparse representation with respect to some discrete basis $W \in \mathbb{R}^{n \times n}$, that is $\mathbf{x}=W \mathbf{c}$, where $\mathbf{c}$ is a sparse signal with $\|\mathbf{c}\|_{0}=k$, $k<n{ }^{1}$ One of the central results of the theory of CS states that a $k$-sparse signal $\mathbf{x}$ can be reliably recovered from $m>k \log (n / k)$ measurements acquired according to

$$
\mathbf{y}=\Psi \mathbf{x}+\mathbf{n},
$$

where $\mathbf{y} \in \mathbb{R}^{m}, m<n$ [13], [14]. Here, $\Psi \in \mathbb{R}^{m \times n}$ is a full-rank matrix known as the sensing matrix and $\mathbf{n}$ denotes measurement noise, which is usually assumed to be additive Gaussian noise.

Finding the sparsifying transform $W$ is a critical task. Thus, for instance, for a broad class of signals, $W$ can be chosen to represent a discrete wavelet transform (DWT), while for other signal classes, $W$ can be obtained using dictionary learning algorithms [24]. It should be noted that, successful signal recovery by means of CS is not a priori guaranteed for an arbitrary choice of matrices $W$ and $\Psi$. For this to happen,

\footnotetext{
${ }^{1}$ Here $\|\cdot\|_{0}$ denotes the $\ell_{0}$-norm that counts the number of non-zero elements of $\mathbf{c}$
}

the matrix $\Phi=\Psi W$ has to obey the restricted isometry property (RIP) of order $k$, which requires

$$
1-\delta_{k} \leq \frac{\|\Phi \mathbf{c}\|_{2}^{2}}{\|\mathbf{c}\|_{2}^{2}} \leq 1+\delta_{k},
$$

to hold $\forall \mathbf{c} \in \mathbb{R}^{n}$, with $\|\mathbf{c}\|_{0}=k$ [13], [14]. Moreover, if this is the case, then a unique approximation $\mathbf{c}$ to the true vector of signal representation coefficients can be obtained through solving the following convex optimization problem

$$
\mathbf{c}=\arg \min _{\mathbf{c}^{\prime}}\left\|\mathbf{c}^{\prime}\right\|_{1}+\lambda\left\|\Phi \mathbf{c}^{\prime}-\mathbf{y}\right\|_{2}^{2}
$$

where $\lambda>0$ is a regularization parameter which depends on the level of noise $\mathbf{n}$. Numerous algorithms have been developed in the literature for solving (3) [25]-[27].

Note that although RIP condition has been used to derive numerous results in the theory of $\mathrm{CS}$, it is only a sufficient condition on $\Phi$, not necessary. That is, cases exist in which a useful approximation to $\mathbf{x}$ in (1) can be obtained with $\Phi$ that does not satisfy RIP. For instance, multiplication of both sides of (1) by an invertible matrix $B$ will clearly have no effect on the set of possible solutions, while the "new" sensing matrix might start violating the RIP. In fact, it can be shown that one can always find such a $B$ which will result in violation of RIP for any sensing matrix [28]-[30]. For the above reasons, we do not use RIP to analyze our results.

In the noiseless case, the feasible region of (3) is defined by $S_{\Phi, \mathbf{y}}=\{\mathbf{c} \mid \Phi \mathbf{c}=\mathbf{y}\}$, which is isomorphic to the nullspace null $(\Phi)$ of $\Phi$, and this is where the optimal solution is located. Thus, intuitively, if the number of measurements $m$ decreases (i.e., the dimension of null( $\Phi)$ increases), the search for the optimal solution would become more challenging and time-consuming. One might therefore suggest that studying null $(\Phi)$ can be rather beneficial in the context of CS. Consequently, using this line of arguments, an alternative approach to the analysis of CS problems have been recently developed, which provides results similar to these obtained based on RIP [28]-[30]. This approach uses the notion of spherical section property. Formally, a subspace $C \subset \mathbb{R}^{n}$ of dimension $\operatorname{dim}(C)=n-m$ is said to have spherical section property (SSP) with distortion $\Delta$ if

$$
\forall \mathbf{c} \neq 0 \in C \rightarrow \frac{\|\mathbf{c}\|_{1}}{\|\mathbf{c}\|_{2}} \geq \sqrt{\frac{m}{\Delta}} .
$$

A small $\Delta$ suggests that the intersection of null $(\Phi)$ and the unit ball looks spherical.

An analogous theorem which uses the notion of SSP states that if null $(\Phi)$ has the $\Delta$-spherical section property and $\mathbf{c}$ is sparse enough, i.e.

$$
\|\mathbf{c}\|_{0} \leq \frac{m}{2 \Delta} \leq \frac{n}{2},
$$

then the classical results of the theory of CS on the uniqueness and stability of signal reconstruction apply [28]-[30]. In addition to CS theorems derived using RIP approach, the SSP-based analysis provides slightly stronger results which allow a deeper insight into the properties of the solution set of (3) under the presence of side information. 
Both RIP and SSP are useful tools to derive CS existential theorems, but unfortunately their direct application in practice is generally complicated, if at all possible. That is, it is computationally intractable to calculate RIP or SSP parameters for an arbitrary matrix $\Phi$ in a general case [28]-[30]. However, computationally tractable bounds for calculating SSP and RIP have been developed in the literature [31], [32]. In the current study we use the upper bounds on SSP to study the effect of adding side information.

Due to the scale invariance of (4) with respect to $\|\mathbf{c}\|_{2}$, $\Delta$ can be found through solving the following problem

$$
\begin{array}{r}
\Delta=\arg \max _{\mathbf{c}}\left\{\|\mathbf{c}\|_{2}^{2}\right\} \\
\text { s.t. } \Phi \mathbf{c}=0 \\
\|\mathbf{c}\|_{1}^{2} \leq 1
\end{array}
$$

Now if we let $C=\mathbf{c c}^{T}$, then, with $I$ being an identity matrix and $\langle B, C\rangle=\operatorname{trace}\left(B C^{T}\right)$ standing for the inner product of matrices $B$ and $C$, (6) can be rewritten in an alternative form as

$$
\begin{gathered}
\Delta=\arg \max _{C}\{\langle C, I\rangle\} \\
\text { s.t. }\left\langle\Phi^{T} \Phi, C\right\rangle=0, \\
\|C\|_{1} \leq 1, \\
\operatorname{Rank}(C)=1,
\end{gathered}
$$

where $\|C\|_{1}=\sum_{i, j} C_{i j}=\|\mathbf{c}\|_{1}^{2}$. By solving (7), one can find an exact value of $\Delta$. However, since the rank constraint is not convex, such a solution is difficult to achieve by using the methods of convex optimization.

The above practical difficulty can be overcome by means of convex relaxation. In particular, the problem in (7) can be convexified by dropping the rank constraint [31]. While amenable to efficient practical treatment, however, the resulting convex minimization problem can be only used to solve for an upper boundary $\bar{\Delta}$ on the SSP distortion parameter $\Delta$. Specifically, $\bar{\Delta}$ can be obtained through

$$
\begin{gathered}
\Delta=\arg \max _{C}\{\langle C, I\rangle\} \\
\text { s.t. }\left\langle\Phi^{T} \Phi, C\right\rangle=0, \\
\|C\|_{1} \leq 1, \\
C \succeq 0 .
\end{gathered}
$$

The problem above is an instance of semidefinite programming (SDP), for which numerous solution methods are currently available [33]. In this paper, we solve (10) with the help of the CVX software package [34].

\section{Proposed Method}

Let $(x, y, z(x, y))$ represent an original 3D surface to be recovered. For the sake of convenience, $z(x, y)$ is assumed to be discretized over a finite-dimensional, uniform, rectangular lattice $\Omega=\{(i, j) \mid 0 \leq i \leq N-1,0 \leq j \leq M-1\} \subset \mathbb{Z}^{2}$, so that the samples of its partial derivatives $\mathbf{z}_{x} \in \mathbb{R}^{n}$ and $\mathbf{z}_{y} \in \mathbb{R}^{n}$ can be concatenated into two column vectors by lexicographic ordering, where $n=M N$. The measurement vectors $\mathbf{b}_{x} \in \mathbb{R}^{m}$ and $\mathbf{b}_{y} \in \mathbb{R}^{m}$ corresponding to $\mathbf{z}_{x}$ and $\mathbf{z}_{y}$, respectively, are obtained as $\mathbf{b}_{x}=\Psi_{x} \mathbf{z}_{x}$ and $\mathbf{b}_{y}=\Psi_{y} \mathbf{z}_{y}$, where $\Psi_{x} \in \mathbb{R}^{m \times n}$ and $\Psi_{y} \in \mathbb{R}^{m \times n}$ denote subsampling matrices which account for the effect of partial observation. It is also assumed that the partial derivatives $\mathbf{z}_{x}$ and $\mathbf{z}_{y}$ admit sparse representations with respect to a linear transformation $W$, which implies the existence of two sparse vectors of representation coefficients $\mathbf{c}_{x}$ and $\mathbf{c}_{y}$ such that $\mathbf{z}_{x}=W \mathbf{c}_{x}$ and $\mathbf{z}_{y}=W \mathbf{c}_{y}$. Finally, it is assumed that both $\operatorname{null}\left(\Psi_{x}\right)$ and null $\left(\Psi_{y}\right)$ satisfy SSP.

Under the above conditions, CS-based reconstruction of the representation coefficients $\mathbf{c}_{x}$ and $\mathbf{c}_{y}$ can be performed in a separable fashion according to

$$
\mathbf{c}_{x}^{*}=\arg \min _{\mathbf{c}_{x}^{\prime}}\left\{\frac{1}{2}\left\|\Psi_{x} W \mathbf{c}_{x}^{\prime}-\mathbf{b}_{x}\right\|_{2}^{2}+\lambda\left\|\mathbf{c}_{x}^{\prime}\right\|_{1}\right\}
$$

and

$$
\mathbf{c}_{y}^{*}=\arg \min _{\mathbf{c}_{y}^{\prime}}\left\{\frac{1}{2}\left\|\Psi_{y} W \mathbf{c}_{y}^{\prime}-\mathbf{b}_{y}\right\|_{2}^{2}+\lambda\left\|\mathbf{c}_{y}^{\prime}\right\|_{1}\right\} .
$$

Moreover, by allowing $\mathbf{c}=\left[\mathbf{c}_{x}^{T}, \mathbf{c}_{y}^{T}\right]^{T}, \mathbf{y}=\left[\mathbf{b}_{x}^{T}, \mathbf{b}_{y}^{T}\right]^{T}$, and $\Phi=\operatorname{diag}\left\{\Psi_{x} W, \Psi_{y} W\right\}$, one can combine (13) and (14) into a single optimization problem as given by

$$
\mathbf{c}^{*}=\arg \min _{\mathbf{c}^{\prime}}\left\{\frac{1}{2}\left\|\Phi \mathbf{c}^{\prime}-\mathbf{y}\right\|_{2}^{2}+\lambda\left\|\mathbf{c}^{\prime}\right\|_{1}\right\} .
$$

This problem is equivalent to (3) and hence any CS solver can be used to find an optimal $\mathbf{c}^{*}$.

Central to DCS is the idea to augment the CS approach in (15) with side information which arises from the fact that, for a twice differentiable surfaces $z(x, y)$, the following relation always takes place [16]

$$
\frac{\partial^{2} z}{\partial x \partial y}=\frac{\partial^{2} z}{\partial y \partial x} \text {. }
$$

This side information can be incorporated in the form of an additional, cross-derivative constraint. In particular, let $D_{x}$ and $D_{y}$ denote the matrices of discrete partial differences in the direction of $x$ and $y$, respectively. Then, the cross-derivative constraint suggests that

$$
D_{x} \mathbf{z}_{y}=D_{y} \mathbf{z}_{x} .
$$

Intuitively the constraint in (17) can be viewed as "additional measurements", whose inclusion can be reasonably expected to improve the accuracy of surface recovery.

To analyze the effect of adding the side information, let $B:=D_{y} W T_{x}-D_{x} W T_{y}$, with $T_{x}$ and $T_{y}$ being the operators of coordinate projections, defined as $\mathbf{c}_{x}=T_{x} \mathbf{c}$ and $\mathbf{c}_{y}=T_{y} \mathbf{c}$. Then, one can amalgamate the actual data and the crossderivative constraint $B \mathbf{c}=0$ into an equivalent measurement model as given by

$$
\mathbf{y}^{\prime}=\Phi^{\prime} \mathbf{c}+\mathbf{n}^{\prime},
$$

where $\Phi^{\prime}:=\left[\Phi^{T}, B^{T}\right]^{T}, \mathbf{y}^{\prime}=\left[\mathbf{y}^{T}, 0^{T}\right]^{T}$, and $\mathbf{n}^{\prime}=\left[\mathbf{n}^{T}, 0^{T}\right]^{T}$. Note that $\Phi^{\prime} \in \mathbb{R}^{(2 m+n) \times 2 n}$ and thus we set $2 m<n$ in order to perform CS on (18).

Since the equivalent measurement model in (18) has the same format as (1), it is reasonable to conclude that the effect of adding the cross-derivative constraint can be assessed 


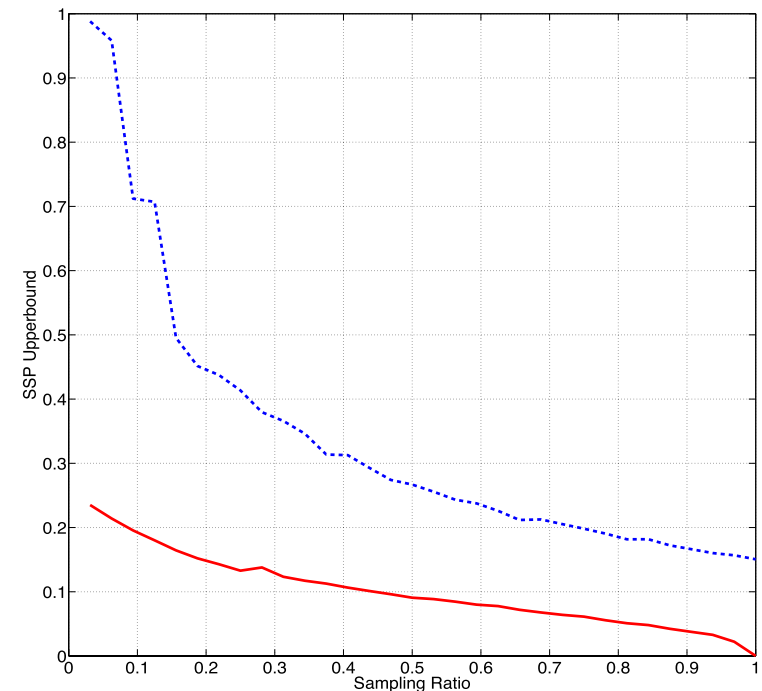

Fig. 1. Upper-bounds on SSP of different sensing matrices as a function of the sampling ratio $(n=32)$. Here, the solid and dashed lines correspond to the cases of DCS and CS, respectively.

through analyzing null $\left(\Phi^{\prime}\right)$. Moreover, as adding the crossderivative constraint decreases the dimension of null $\left(\Phi^{\prime}\right)$, it can be expected to decrease the distortion of the sensing matrix $\Phi^{\prime}$. Consequently, (5) suggests that, for a fixed error rate, the same signal c could be recovered from a smaller number of measurements $2 m$. Alternatively, keeping the number of measurements $2 m$ fixed, one could expect to successfully recover signals characterized by a lower degree of sparsity.

The above intuitive considerations can be further supported in a more formal way as follows. First, we note that

$$
\operatorname{null}\left(\Phi^{\prime}\right)=\operatorname{null}(\Phi) \cap \operatorname{null}(B) \rightarrow \operatorname{null}\left(\Phi^{\prime}\right) \subset \operatorname{null}(\Phi) .
$$

Thus, since null $(\Phi)$ satisfies SSP with parameters $m$ and $\Delta, \operatorname{null}\left(\Phi^{\prime}\right)$ should also satisfy SSP with (at least) the same parameters. Equivalently, it follows from (4), that $m^{\prime}=2 m+n$ and $\Delta^{\prime} \leq \Delta(1+n / m)$, while $m^{\prime} / \Delta^{\prime}=m / n$. Now, since (18) has a unique solution and any feasible solution of (1) satisfies (18) as well, we conclude that (18) should share the same unique solution with (1). This fact ensures that adding the cross-derivative constraint is unlikely to result in a violation of the uniqueness of the solution to (1). However, to validate the beneficial effect of incorporating this additional constraint, we need to check whether or not it leads to a reduced distortion of the new sensing matrix $\Phi^{\prime}$.

In this work, the above question is addressed through a numerical experiment. To this end, for $n=32$, we let $\Phi$ be a random matrix with i.i.d. entries drawn from a normal distribution, while $W$ is defined to be an overcomplete discrete cosine transform dictionary [35]. Subsequently, we use (7) to estimate the upper-bounds on SSP for the sensing matrices $\Phi$ and $\Phi^{\prime}$ as a function of the sampling ratio $r=m / n$. The results of our simulations are depicted in Fig. 1. Note that here we have plotted $\frac{\Delta}{m}$ because according to (4) and (5), normalized $\Delta$ must be compared. This shows an expected behavior with $\bar{\Delta}_{\Phi^{\prime}} \leq \bar{\Delta}_{\Phi}$. Thus, this numerical example can be viewed as an empirical confirmation of the fact that adding the cross-derivative constraint indeed holds potential to improve the accuracy of signal reconstruction.
Algorithm 1 Derivative Compressive Sampling

1) Data: $\mathbf{b}_{x}, \mathbf{b}_{y}$, and $\lambda>0$.

2) Initialization: For a given transform matrix $W$ and matrices/operators $\Psi_{x}, \Psi_{y}, D_{x}, D_{y}, T_{x}$ and $T_{y}$, preset the procedures of multiplication by $\Phi, \Phi^{T}, B$ and $B^{T}$.

3) Gradient field recovery: Starting with an arbitrary $\mathbf{c}^{(0)}$ and $p^{(0)}=0$, iterate (21) until convergence to result in an optimal $\mathbf{c}^{*}$. Use the estimated partial derivatives $W T_{x} \mathbf{c}^{*}$ and $W T_{y} \mathbf{c}^{*}$ to recover the values of $\mathbf{z}_{x}$ and $\mathbf{z}_{y}$.

4) Source recovery: Use a Poisson solver to reconstruct the original source from its gradient field.

Next, in order to solve the problem (18) an efficient numerical procedure is required. Clearly, to this end, one can just use a standard CS solver. However, it is possible to further improve the numerical efficiency of the reconstruction by taking advantage of the specific nature of the cross-derivative constraint. Specifically, the problem at hand can be formulated as a constrained optimization problem of the form

$$
\begin{aligned}
\mathbf{c}^{*}= & \arg \min _{\mathbf{c}^{\prime}}\left\{\frac{1}{2}\left\|\Phi \mathbf{c}^{\prime}-\mathbf{y}\right\|_{2}^{2}+\lambda\left\|\mathbf{c}^{\prime}\right\|_{1}\right\}, \\
& \text { s.t. } B \mathbf{c}^{\prime}=0 .
\end{aligned}
$$

This is a classical optimization problem, which can be efficiently solved by the augmented Lagrangian method [36], which finds the optimal solution via a series of iterations computed according to

$$
\begin{aligned}
& \mathbf{c}^{(t+1)}=\arg \min _{\mathbf{c}^{\prime}}\left\{\frac{1}{2}\left\|\Phi \mathbf{c}^{\prime}-\mathbf{y}\right\|_{2}^{2}+\lambda\left\|\mathbf{c}^{\prime}\right\|_{1}+\frac{\delta}{2}\left\|B \mathbf{c}^{\prime}+p^{(t)}\right\|_{2}^{2}\right\} \\
& p^{(t+1)}=p^{(t)}+B \mathbf{c}^{(t+1)},
\end{aligned}
$$

where $p^{(t)}$ is a (dual) vector of (weighted) Lagrange multipliers, $\delta>0$ is a penalty parameter, and $t$ stands for the iteration index. Note that in (21), the update of $\mathbf{c}^{(t+1)}$ has the format of a lasso problem [37] which can be solved by a variety of computational algorithms. In this work, the solution has been obtained by means of the fast iterative shrinkage thresholding algorithm (FISTA) of Beck et al [25].

Given the optimal solution $\mathbf{c}^{*}$, the full measurement set of partial derivatives can be recovered as $\mathbf{z}_{x}=W T_{x} \mathbf{c}$ and $\mathbf{z}_{y}=W T_{y} \mathbf{c}$, which, in turn, can be used to approximate the original surface $z(\cdot, \cdot)$ via solution of a Poisson equation [17]. Algorithm 1 above summarizes all the computational steps of the proposed algorithm.

\section{Surface Reconstruction in Gradient Field}

The problem of surface reconstruction is a standard problem in computer vision, where the goal is to use multiple 2D images to recover a close approximation of a 3D surface, $z(x, y)$. To simplify hardware design, it is usually found convenient to reconstruct the surface from its orientations in the $2 \mathrm{D}$ gradient space $\left(z_{x}, z_{y}\right)$, while in practice, the gradient field is determined through a reflectance map $R\left(z_{x}, x_{y}\right)$ [7], which, in turn, is measured empirically. Note that the reflectance map can be viewed as a $2 \mathrm{D}$ image $i(x, y)$, with the image intensities being a function of $z_{x}$ and $z_{y}$. 
For a surface $z(x, y)$, the normal vector can be computed as $\left[z_{x}, z_{y},-1\right]$, thus we can explicitly represent a surface in gradient space $\left(z_{x}, z_{y}\right)$. Because the ratio of the light reflected from a surface depends on surface gradient [7], a class of imaging devices measure surface gradients by computing reflectance map. A simple but, at the same time, the most conventional model used to describe the observation of $3 \mathrm{D}$ surfaces is the Lambertian model [7], in which the light reflected in a given direction depends predominantly on the surface orientation. Specifically, if the measuring camera is placed at infinity (a single distant point source) for which the perspective projection can be approximated as orthographic projection, the reflectance map based on the Lambertian shading rule is defined as given by [7]

$$
R\left(z_{x}, z_{y}\right)=\frac{\rho\left(1+z_{x} p_{s}+z_{y} q_{s}\right)}{\sqrt{1+z_{x}^{2}+z_{y}^{2}} \sqrt{1+p_{s}^{2}+q_{y}^{s}}}
$$

where $\rho$ is a reflectance coefficient, and $p_{s}$ and $q_{s}$ determine the direction of light source.

Equation (22) can be used to to determine surface orientation from measured intensity. But it has nonlinearity and given $R\left(z_{x}, z_{y}\right)$, we have two unknowns $z_{x}$ and $z_{y}$. A basic idea behind both photometric stereo (PS) [7] and shape-fromshading (SFS) [8] is to gradually alter the viewing direction to measure the $x$ and $y$ components of the gradient field of a surface, $z(x, y)$, followed by integrating these measurements into a $3 \mathrm{D}$ surface reconstruction. Thus, for a known $\rho$, at least two views are required for determining $z_{x}$ and $z_{y}$. However, due to the nonlinear nature of (22), such a solution would not be unique, in general. To alleviate this deficiency, at least three measurements with three different light directions are required to solve uniquely for $z_{x}$ and $z_{y}$. In practice, for improving the robustness of surface reconstruction towards the effect of measurement noise, it is standard to use $N$ images $i(x, y)=R\left(z_{x}, z_{y}\right)$, with $(N>3)$ [38]. Subsequently, for each point $\left(x_{i}, x_{j}\right)$, these images are used to form the following system of equations

$$
\left[\begin{array}{c}
i_{1}(i, j) \\
\vdots \\
i_{N}(i, j)
\end{array}\right]=\left[\begin{array}{ccc}
d_{1 x} & d_{1 y} & d_{1 z} \\
\vdots & \vdots & \vdots \\
d_{N x} & d_{N y} & d_{N z}
\end{array}\right]\left[\begin{array}{c}
\hat{n}_{x} \\
\hat{n}_{y} \\
\hat{n}_{z}
\end{array}\right],
$$

where $\left(d_{k x}, d_{k y}, d_{k z}\right)$ denotes the $k^{t h}$ direction of the light ray (viewing point) and $\hat{\mathbf{n}}^{T}=\left[\hat{n}_{x}, \hat{n}_{y}, \hat{n}_{z}\right]^{T}$ is the surface normal vector. In a matrix form, this equation can be written as

$$
I=D \hat{\mathbf{n}},
$$

which admits a closed-form solution in terms of the Moore-Penrose inverse of $D$, viz.

$$
\hat{\mathbf{n}}=\left(D^{T} D\right)^{-1} D^{T} I,
$$

Note that $\hat{\mathbf{n}}$ is a unit vector which is parallel to $\left[z_{x}, z_{y},-1\right]$ $\left(\left[z_{x}, z_{y},-1\right] \propto\left[\hat{n}_{x}, \hat{n}_{y}, \hat{n}_{z}\right]\right)$. Thus, having the unit surface normal vector estimated, the $x$ and $y$ components of the gradient field can be computed as

$$
\begin{aligned}
z_{x} & =\hat{n}_{x} / \hat{n}_{z}, \\
z_{y} & =\hat{n}_{y} / \hat{n}_{z} .
\end{aligned}
$$

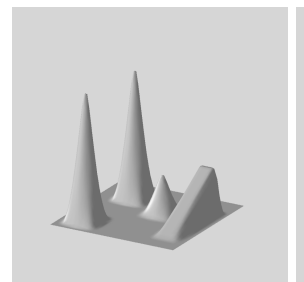

(a)

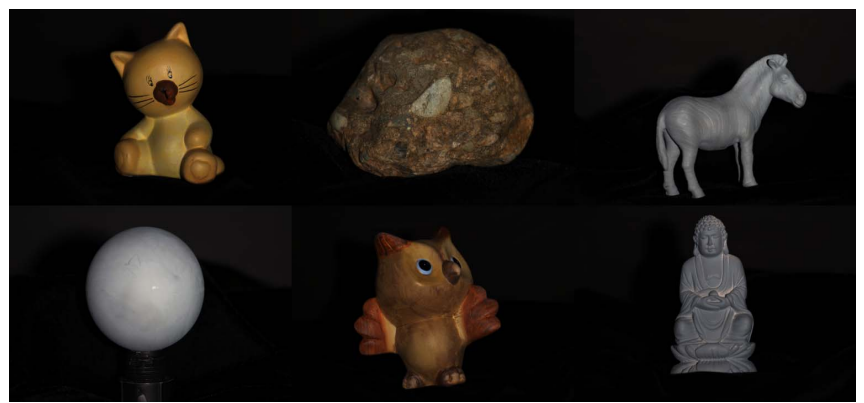

(d)

Fig. 2. Synthetic surfaces (a) Ramp-peak (b) Sphere, (c) Peak-valley, and (d) sample photos of 6 real objects. (Top from left to right: Cat, Rock, and Horse. Bottom from left to right: Gray, Owl, and Buddha.)

Finally, the resulting estimates of the partial derivatives can be arranged into two numeric arrays defined as

$$
\begin{aligned}
& Z_{x}(i, j)=\left.\frac{\partial z}{\partial x}\right|_{(x, y)=\left(x_{i}, y_{j}\right)}, \\
& Z_{y}(i, j)=\left.\frac{\partial z}{\partial y}\right|_{(x, y)=\left(x_{i}, y_{j}\right)} .
\end{aligned}
$$

In practice, an accurate surface reconstruction necessitates using a relatively large number of sampling points, which may lead to prohibitive acquisition requirements from the viewpoint of hardware design and its practical limitations [12]. Using less dense sampling sets, on the other hand, could result in suboptimal reconstructions of poor quality [7]. However, this unfortunate situation can be greatly improved through the use of DCS. In this case, given partial measurements of $Z_{x}$ and $Z_{y}$, one can employ Algorithm 1 to solve for $\mathbf{z}_{x}$ and $\mathbf{z}_{y}$. This step can be interpreted as increasing the sampling density of the gradient field (without complicating the hardware design). Note, in the final stage of Algorithm 1, a surface shape is recovered via solving a Poisson equation under proper boundary conditions, which can be implemented in a variety of ways [8]-[10], [39], [40]. In this work, we take advantage of the spectral (i.e., frequency-domain) method of [10].

\section{EXPERIMENTAL RESULTS}

In the experimental part of this work, we compared the performance of the proposed DCS method against: (a) classical $\mathrm{CS}$ and (b) reconstruction based on dense sampling (DS). In our experiments, we used the synthetic surfaces from [41] and [42] (see Fig. 2 (a)-(c)) as well as real world measurements from six different objects (see Fig. 2 (d)) 
TABLE I

Comparisons of Surface Recovery Results for Different MEASUREMENT Noise LeVels With $r=0.5$

\begin{tabular}{|c|c|c|c|c|c|c|c|c|c|c|c|c|}
\hline SNR (dB) & 10 & 15 & 20 & 25 & 10 & 15 & 20 & 25 & 10 & 15 & 20 & 25 \\
\hline Surface & \multicolumn{4}{|c|}{ Ramp-Peak } & \multicolumn{4}{|c|}{ Sphere } & \multicolumn{4}{|c|}{ Peak-Valley } \\
\hline$\overline{\mathrm{DS}}$ & 31.49 & 36.35 & 40.97 & 41.32 & 15.54 & 15.66 & 15.59 & 15.57 & 19.90 & 19.58 & 19.62 & 19.63 \\
\hline CS & 19.19 & 19.90 & 22.13 & 23.07 & 10.11 & 10.38 & 10.22 & 10.19 & 10.97 & 9.27 & 10.86 & 10.62 \\
\hline DCS & 27.55 & 30.59 & 33.55 & 35.44 & 10.51 & 10.75 & 10.72 & 10.61 & 14.40 & 13.27 & 13.96 & 14.01 \\
\hline Surface & \multicolumn{4}{|c|}{ Cat } & \multicolumn{4}{|c|}{ Rock } & \multicolumn{4}{|c|}{ Horse } \\
\hline$\overline{\mathrm{DS}}$ & 33.36 & 37.54 & 42.91 & 48.25 & 34.99 & $\overline{41.48}$ & $\overline{46.00}$ & 51.05 & 29.43 & 34.62 & 39.54 & 45.22 \\
\hline $\mathrm{CS}$ & 10.49 & 10.12 & 9.90 & 10.79 & 11.53 & 11.38 & 12.33 & 12.10 & 9.23 & 8.37 & 8.92 & 9.11 \\
\hline DCS & 25.64 & 29.03 & 32.40 & 32.84 & 27.49 & 30.81 & 31.39 & 33.07 & 21.20 & 23.32 & 28.40 & 27.85 \\
\hline Surface & \multicolumn{4}{|c|}{ Gray } & \multicolumn{4}{|c|}{ Owl } & \multicolumn{4}{|c|}{ Buddha } \\
\hline DS & 41.37 & 44.94 & 49.67 & 50.63 & 34.04 & 39.04 & 43.95 & 49.12 & 31.91 & 36.82 & 42.04 & 45.80 \\
\hline $\mathrm{CS}$ & 16.20 & 18.03 & 18.82 & 19.35 & 11.30 & 11.64 & 12.45 & 12.76 & 10.57 & 11.09 & 10.90 & 11.36 \\
\hline DCS & 30.63 & 37.56 & 40.55 & 42.95 & 27.40 & 31.38 & 36.42 & 38.98 & 23.64 & 29.90 & 31.68 & 35.81 \\
\hline
\end{tabular}

TABLE II

Comparisons of SuRface ReCovery Results for DifFERENT OUtLIERs LEVELS $\left(d_{o}\right)$ With SNR $=20 \mathrm{~dB}$

\begin{tabular}{|c|c|c|c|c|c|c|c|c|c|c|c|c|}
\hline Outliers level $\left(d_{o}\right)$ & 0.04 & 0.06 & 0.08 & 0.1 & 0.04 & 0.06 & 0.08 & 0.1 & 0.04 & 0.06 & 0.08 & 0.1 \\
\hline Surface & \multicolumn{4}{|c|}{ Ramp-Peak } & \multicolumn{4}{|c|}{ Sphere } & \multicolumn{4}{|c|}{ Peak-Valley } \\
\hline DS & 24.04 & 22.19 & 21.93 & 19.49 & 18.86 & 17.64 & 15.15 & 14.34 & 22.34 & 17.33 & 16.61 & 15.66 \\
\hline CS & 12.51 & 11.99 & 10.12 & 10.04 & 13.01 & 11.23 & 10.19 & 9.98 & 14.43 & 12.96 & 11.35 & 9.20 \\
\hline DCS & 20.81 & 19.20 & 18.43 & 17.35 & 15.67 & 14.34 & 13.53 & 12.28 & 21.95 & 16.93 & 15.52 & 14.10 \\
\hline Surface & \multicolumn{4}{|c|}{ Cat } & \multicolumn{4}{|c|}{ Rock } & \multicolumn{4}{|c|}{ Horse } \\
\hline DS & 18.53 & 17.50 & 16.60 & 16.14 & 22.96 & 20.26 & 19.02 & 18.37 & 16.77 & 15.01 & 13.26 & 12.39 \\
\hline $\mathrm{CS}$ & 12.23 & 11.61 & 10.96 & 10.54 & 13.31 & 13.22 & 12.67 & 12.15 & 11.67 & 10.31 & 9.67 & 10.26 \\
\hline DCS & 15.53 & 14.63 & 13.17 & 12.10 & 15.81 & 15.72 & 14.92 & 13.38 & 15.08 & 14.96 & 13.70 & 12.06 \\
\hline Surface & \multicolumn{4}{|c|}{ Gray } & \multicolumn{4}{|c|}{ Owl } & \multicolumn{4}{|c|}{ Buddha } \\
\hline$\overline{\mathrm{DS}}$ & 23.35 & 22.65 & 22.30 & 21.19 & 27.34 & 26.95 & 23.89 & 21.60 & 25.38 & 23.34 & 22.66 & 21.87 \\
\hline $\mathrm{CS}$ & 15.23 & 13.42 & 12.42 & 11.49 & 20.48 & 19.22 & 18.87 & 15.31 & 14.24 & 12.07 & 11.45 & 10.98 \\
\hline DCS & 19.17 & 18.35 & 17.48 & 16.58 & 24.00 & 23.24 & 21.84 & 20.67 & 22.65 & 21.64 & 20.31 & 18.05 \\
\hline
\end{tabular}

TABLE III

COMPARISONS OF SURFACE RECOVERy RESUltS For DifFERENT SAMPLing RATIOS With SNR $=20 \mathrm{~dB}$

\begin{tabular}{|c|c|c|c|c|c|c|c|c|c|c|c|c|}
\hline Sampling Ratio & 0.2 & 0.4 & 0.6 & 0.8 & 0.2 & 0.4 & 0.6 & 0.8 & 0.2 & 0.4 & 0.6 & 0.8 \\
\hline Surface & \multicolumn{4}{|c|}{ Ramp-Peak } & \multicolumn{4}{|c|}{ Sphere } & \multicolumn{4}{|c|}{ Peak-Valley } \\
\hline $\mathrm{CS}$ & 9.26 & 10.25 & 20.99 & 31.63 & 7.06 & 10.74 & 15.47 & 18.40 & 4.79 & 10.2 & 14.40 & 21.93 \\
\hline DCS & 23.20 & 28.71 & 33.51 & 35.81 & 8.13 & 12.82 & 16.32 & 18.88 & 6.62 & 17.61 & 20.67 & 23.09 \\
\hline Surface & \multicolumn{4}{|c|}{ Cat } & \multicolumn{4}{|c|}{ Rock } & \multicolumn{4}{|c|}{ Horse } \\
\hline CS & 4.86 & 7.65 & 11.59 & 18.25 & 6.38 & 8.87 & 13.44 & 20.75 & 3.30 & 5.94 & 8.88 & 16.19 \\
\hline DCS & 8.00 & 18.52 & 30.30 & 35.05 & 10.99 & 20.33 & 33.13 & 37.42 & 6.01 & 16.82 & 24.40 & 31.07 \\
\hline Surface & \multicolumn{4}{|c|}{ Gray } & \multicolumn{4}{|c|}{ Owl } & \multicolumn{4}{|c|}{ Buddha } \\
\hline $\mathrm{CS}$ & 6.80 & 11.20 & 18.49 & 28.86 & 5.55 & 8.44 & 13.18 & 18.00 & 4.35 & 7.66 & 11.29 & 16.44 \\
\hline DCS & 13.27 & 27.37 & 41.38 & 43.94 & 10.90 & 20.68 & 31.68 & 36.40 & 7.62 & 21.73 & 28.41 & 33.80 \\
\hline
\end{tabular}

(provided by courtesy of Dan Goldman and Steven Seitz ${ }^{2}$ ). The subsampling matrices $\Psi_{x}$ and $\Psi_{y}$ were obtained from an identity matrix $I$ through a random subsampling of its rows by a factor $r$, resulting in a required partial sampling ratio. For sparse representation, $W$ was defined to represent a four-level orthogonal wavelet transform using the nearly symmetric wavelets with five vanishing moments [43]. Experiments confirm that natural surfaces can be represented sparsely in this basis [44]. To compare the reconstruction quality, we have used Signal-to-Noise Ratio, defined as $S N R=10 \log \left(\frac{\sum_{i=0}^{N-1} \sum_{j=0}^{M-1} \mathbf{z}(i, j)^{2}}{\sum_{i=0}^{N-1} \sum_{j=0}^{M-1}(\mathbf{z}(i, j)-\hat{\mathbf{z}}(i, j))^{2}}\right)$, where $\hat{\mathbf{z}}(\cdot, \cdot)$ denotes the reconstructed surface.

Table I to Table IV summarize reconstruction results for different methods, obtained for both synthetic and real world

\footnotetext{
${ }^{2}$ http://courses.cs.washington.edu/courses/csep576/05wi//projects/project3/ project3.htm
}

TABLE IV

COMPARISONS OF SURFACE RECOVERY RESULTS FOR DIFFERENT DECIMATION FACTOR $\left(d_{f}\right)$ With SNR $=20 \mathrm{~dB}$

\begin{tabular}{l|cc|ccc|cc}
\hline \hline Decimation $\left(d_{f}\right)$ & 0.25 & 0.5 & \multicolumn{2}{c|}{0.25} & 0.5 & 0.25 & 0.5 \\
\hline Surface & \multicolumn{2}{|c|}{ Ramp-Peak } & \multicolumn{2}{c}{ Sphere } & \multicolumn{2}{c}{ Peak-Valley } \\
\hline \hline CS & 3.82 & 10.25 & 4.99 & 11.63 & 7.06 & 10.74 \\
DCS & 13.20 & 28.71 & 15.51 & 25.81 & 11.13 & 18.82 \\
\hline \hline Surface & \multicolumn{2}{|c|}{ Cat } & \multicolumn{2}{c}{ Rock } & \multicolumn{2}{c}{ Horse } \\
\hline \hline CS & 4.86 & 7.65 & 8.59 & 11.25 & 6.38 & 8.87 \\
DCS & 10.00 & 18.52 & 16.30 & 25.05 & 10.99 & 20.33 \\
\hline \hline Surface & \multicolumn{2}{|c|}{ Gray } & \multicolumn{2}{c}{ Owl } & \multicolumn{2}{c}{ Buddha } \\
\hline \hline CS & 6.80 & 11.20 & 8.49 & 12.86 & 5.55 & 9.44 \\
DCS & 13.27 & 27.37 & 15.38 & 21.94 & 10.90 & 20.68 \\
\hline
\end{tabular}

data for different partial sampling ratios and noise levels. In particular, Table I summarizes the results for different measurement noise levels and partial sampling ratio of $r=0.5$. 


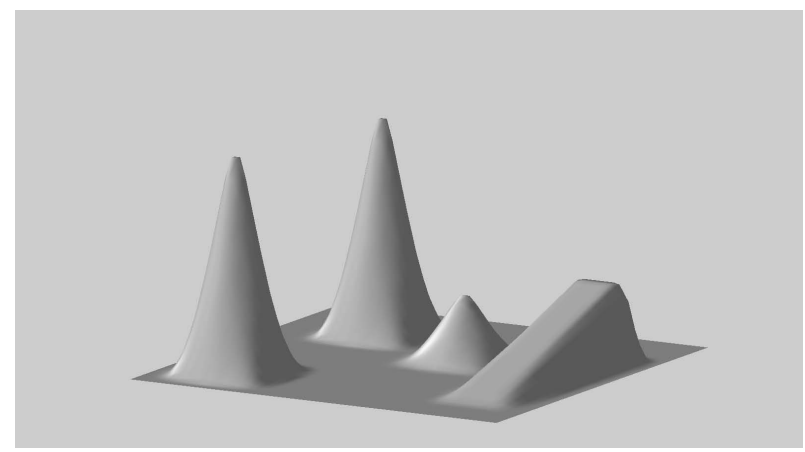

(a)

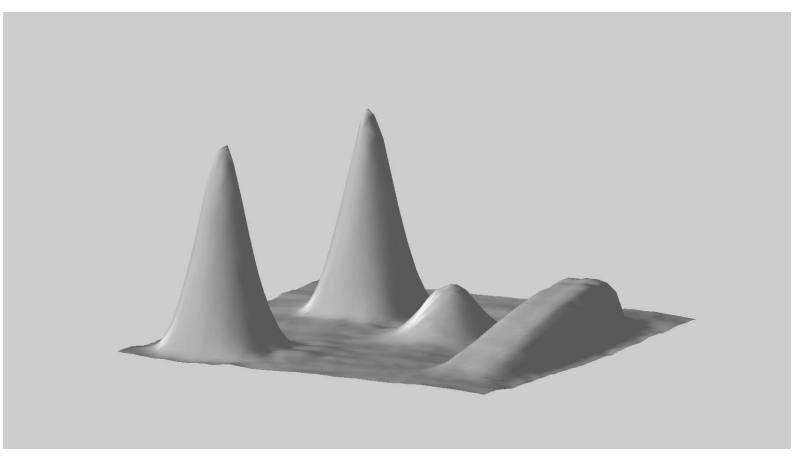

(c)

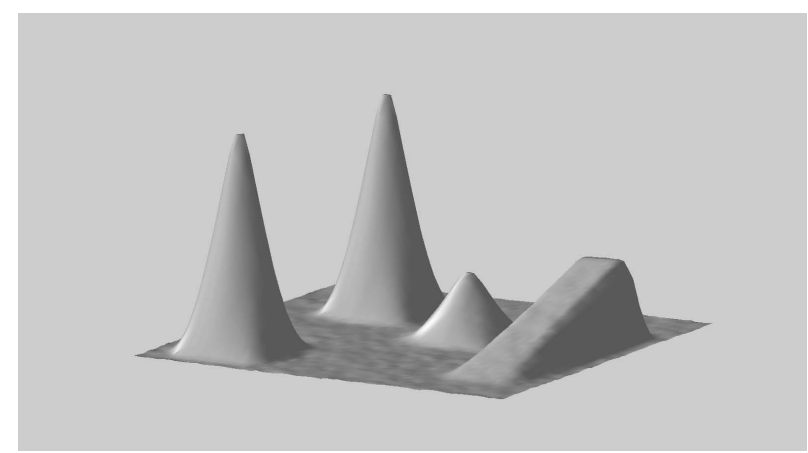

(b)

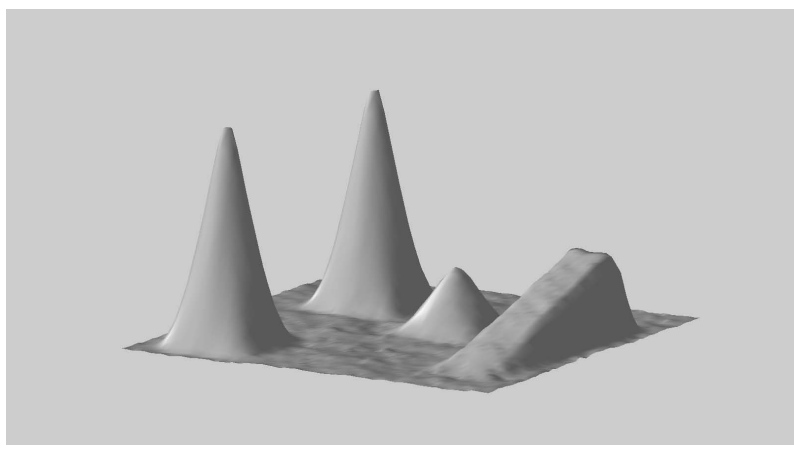

(d)

Fig. 3. Peak-Ramp surface (a) and its reconstructed versions using (b) DS, (c) classical CS, and (d) DCS for SNR $=20$ dB.

Analyzing the table, we first note that, in all cases, dense sampling improves SNR. This reflects the fact that imposing sparsifying priors on the source signal in wavelet domain can be used as a denoising method for surface reconstruction. At the same time, one can see that using CS substantially degrades reconstruction quality, while DCS improves the quality in comparison with CS, providing results comparable to those produced by DS for higher levels of noise. This is an important observation that implies that DCS can be beneficial for reducing the complexity (and hence the cost) of hardware design. Additionally, for the sake of visual comparison, the reconstruction results for the Ramp-Peak synthetic surface and the horse object are given in Fig. 3 and Fig. 4, respectively for SNR $=20 \mathrm{~dB}$. In both cases, one can see that DCS yields reconstructions of nearly the same quality as those computed through DS, while resulting in much less severe artifacts as compared to the case of CS (especially in vicinity of surface edges). As it can also be seen that CS-based reconstructions are characterized by overly smoothed edges - the defect virtually undetectable in the case of the proposed DCS approach.

Note that from the viewpoint of statistical estimation theory, CS asserts a Gaussian noise model, but in practice gradient measurements would be corrupted by both noise and outliers which. In fact, this is considered to be a more standard model [2], [3]. The use of the additional derivative constraints by DCS makes our reconstruction robust toward the inconsistency in noise modeling because these constraints are independent from noise model. This argument is supported by the results in Table II. Here, we have used salt and pepper noise to model outliers [3] in presence of Gaussian noise with
SNR $=20 \mathrm{~dB}$. The parameter $d_{o}$ indicates the proportion of pixels which are missing due to outliers. One can see that, in this case, although reconstruction quality decreases compared to Gaussian noise model, DCS is more robust compared to CS and still can lead to a reconstruction accuracy comparable with dense sampling.

To investigate the performance of the proposed algorithm at different measurement noise regimes, the surface reconstructions have been computed for a range of SNR values. The results of these computations for Ramp-Peak surface are summarized in Fig. 5(a). Clearly, the cross-derivative constraint exploited by DCS effectively restricts the feasibility region for an optimal solution, and thus the algorithm results in a substantially improved reconstruction quality as compared to the case of CS for all SNR values. Note that, even at $\mathrm{SNR}=8 \mathrm{~dB}$, the reconstruction quality of DCS is virtually as good as that of CS reconstructions obtained at $\mathrm{SNR}=30 \mathrm{~dB}$. This is a considerable improvement as compared to CS, which shows that DCS remains a reliable estimation approach even at high noise levels. Although reconstruction results for Ramp-Peak surface may seem convincing, one can argue that these results can be model dependent. In Fig. 5(b), reconstruction results are summarized across the dataset of Fig. 2. Here we have depicted improvement in SNR, that is the difference between the DCS based reconstruction result and the CS based reconstruction result. It can be seen in Table I and Table II that CS reconstruction can vary for different surfaces. We use the SNR difference between CS based and DCS based reconstructions, because CS based reconstruction can be considered as a baseline for comparison. The curve in Fig. 5(b) is the average improvement for the 9 surfaces and the error bars 


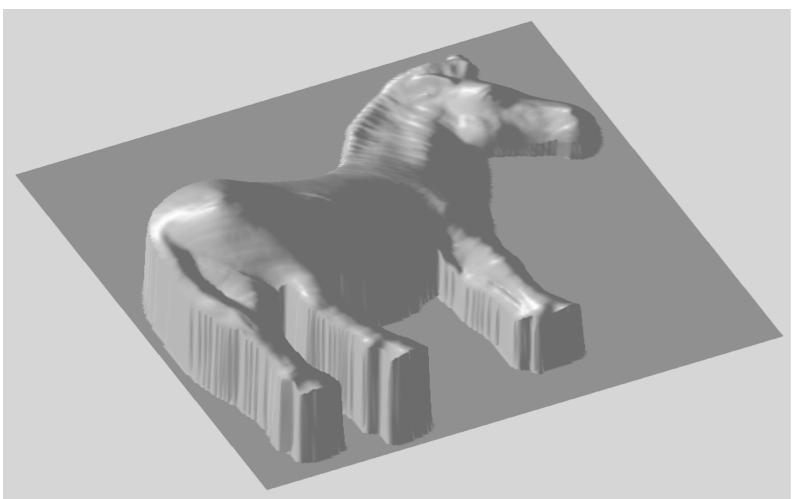

(a)

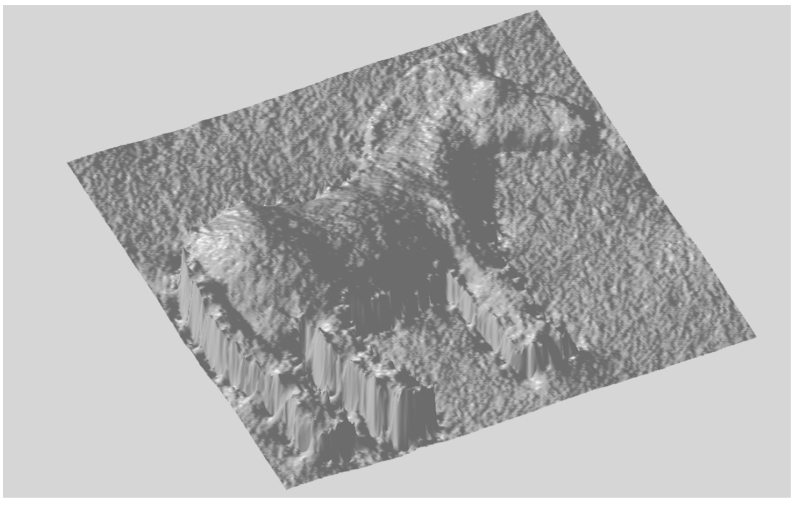

(c)

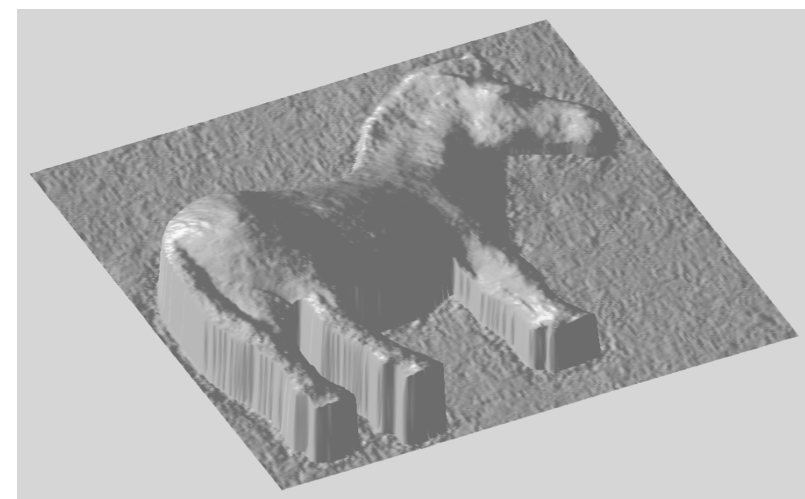

(b)

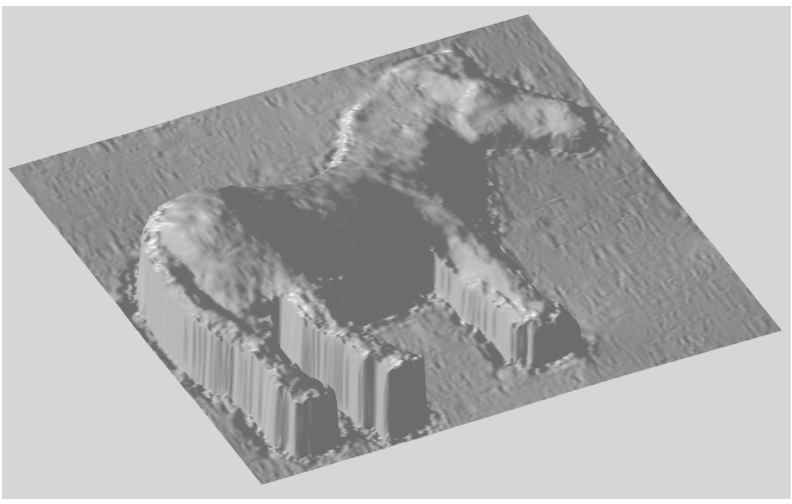

(d)

Fig. 4. Horse surface (a) and its reconstructed versions using (b) DS, (c) classical CS, and (d) DCS for SNR $=20 \mathrm{~dB}$.

denotes variation across the dataset. As it can be seen, a similar trend can be seen across the dataset. Although, the amount of improvement is model dependent and can vary for different surfaces, but error bars confirm that the trend is the dataset.

Table III summarizes the reconstruction results obtained with CS and DCS for different subsampling ratios and a fixed noise level of $\mathrm{SNR}=20 \mathrm{~dB}$. One can see that, once again, DCS outperforms CS for all the cases under comparison. This conclusion is further confirmed by Fig. 6(a) which presents the results of the reconstruction of Ramp-Peak surface obtained for a continuous range of subsampling ratios. This result deserves a special attention, as it shows that the DCS-based reconstruction with $r=0.3$ is comparable with the CS-based reconstruction with $r=0.6$. This is an important observation which suggests that subcritical non-uniform sampling can be used in conjunction with DSC to substantially improve the effective resolution of the hardware in use. To investigate the consistency of the results, in Fig. 6(b), reconstruction results are summarized across the dataset similar to Fig. 5(b). Again, it can be seen that although the amount of improvement is dependent on the surface of interest but error bars confirm that the trend is consistent across the dataset. This observation indicates that, the developed method can be used for surface reconstruction of different source signals.

For these results we have assumed a random sampling procedure to guarantee that the required condition for CS recovery holds. One can argue that, in practice the sampling pattern is decimation because we may have access to low resolution versions of the surface of interest. For this reason, results for uniform decimation pattern are presented in Table IV. We have used two decimation factors $\left(d_{f}\right)$, defined as the downsampling proportion. First, we assume a uniform downsampling in just one spatial direction which leads to $d_{f}=0.5$ and next we assume a uniform decimation in both spatial directions, which gives $d_{f}=0.25$. Reconstruction results confirm that although reconstruction quality decreases when we use decimation sampling, DCS still can lead to much better accuracy.

As an additional comparison, Fig. 7(a) illustrates the convergence of the mean square error (MSE) of residual, defined as $\left\|\mathbf{y}-\Phi \mathbf{c}^{t}\right\|_{2}^{2}$ for each iterations of Algorithm, as a function of the number of iterations, for both CS and DCS algorithms. Furthermore, Fig. 7(b) summarizes MSE of residual across the dataset. Here the curves denote the average performance across dataset and the shaded area denotes the variation across the dataset. Fig. 7(b) in fact shows that convergence of CS is slower than DCS across the dataset, which can be seen as an additional practical advantage offered by the proposed solution. This advantage can help with online surface reconstruction [45], where we are interested in reconstructing a surface over a time interval. In such cases, the speed of reconstruction is crucial to be able to detect surface changes.

\section{LIMITATIONS AND DISCUSSION}

In the present paper, the applicability of DCS to the problem of surface reconstruction has been studied. The proposed DCS-based solution was shown to be capable 


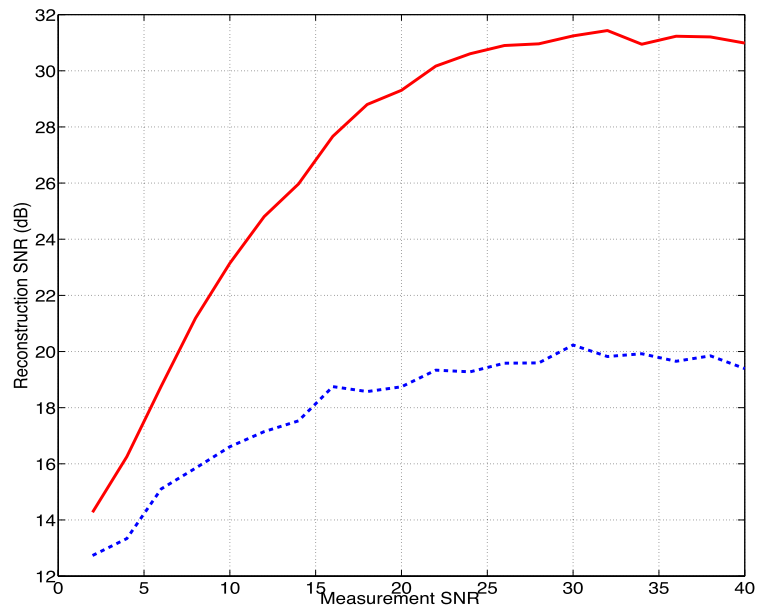

(a)

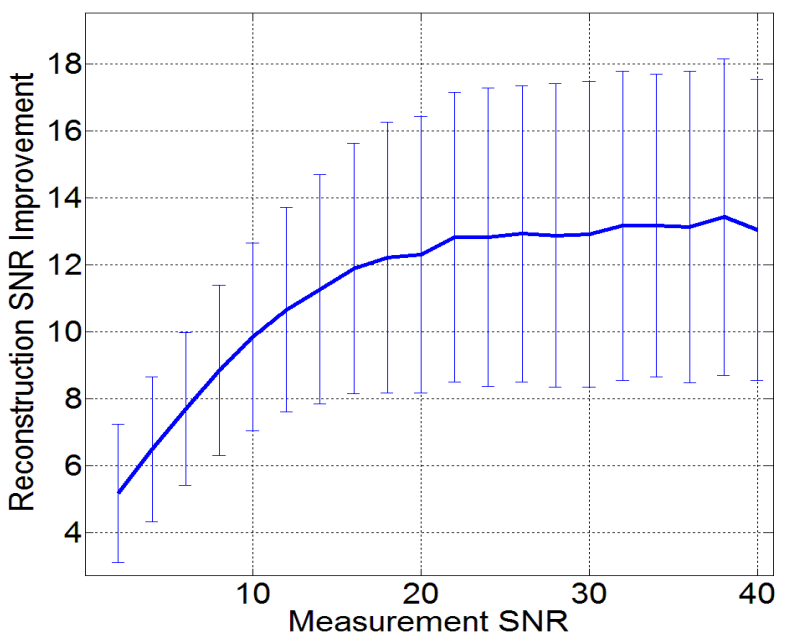

(b)

Fig. 5. Subplot (a) SNR of Ramp-Peak surface reconstruction as a function of SNR for $r=0.5$. Here, the dashed and solid lines correspond to classic CS and DCS, respectively, and $r=0.5$. Subplot (b) Reconstruction SNR improvement as a function of SNR across the dataset of Fig. 2. Here the curve depicts the average improvement of reconstruction results across the dataset and error bars denote the variation across the dataset.

of either simplifying the hardware design or increasing its effective spatial resolution. The proposed method applies CS for surface reconstruction subject to an additional constraint, which stems from some intrinsic properties of gradient fields. Based on the SSP paradigm, in fact, DCS is shown to be better only empirically. Even the value of $\Delta$ is shown to improve only empirically with respect to a classing CS setting. Experiments involving both synthetic surfaces and real world data confirmed the usefulness and effectiveness of the proposed resurface reconstruction approach. It was shown that the DCS-based estimation with $r=0.3$ results in reconstructions of the quality comparable to that of CS with $r=0.6$ for fixed noise level of SNR $=20 \mathrm{~dB}$. Moreover, DCS reconstructions obtained at higher noise levels with $r=0.5$ are comparable with CS reconstructions obtained

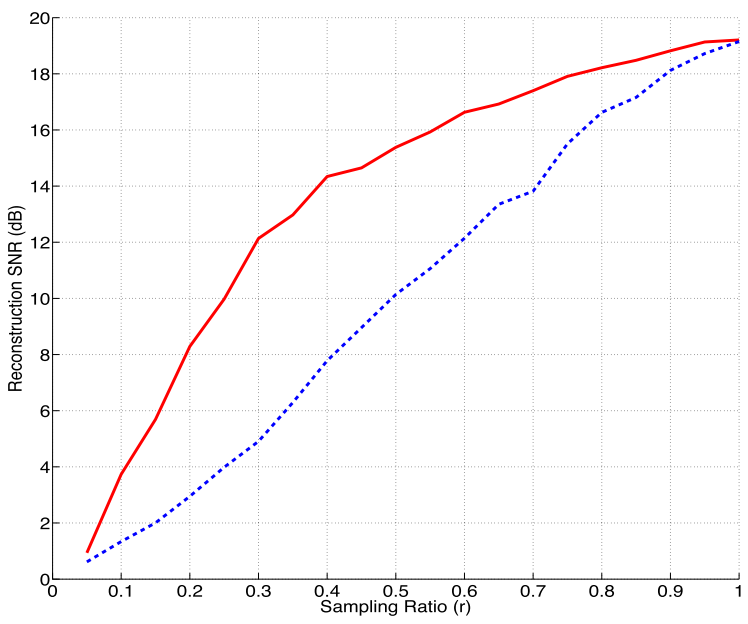

(a)

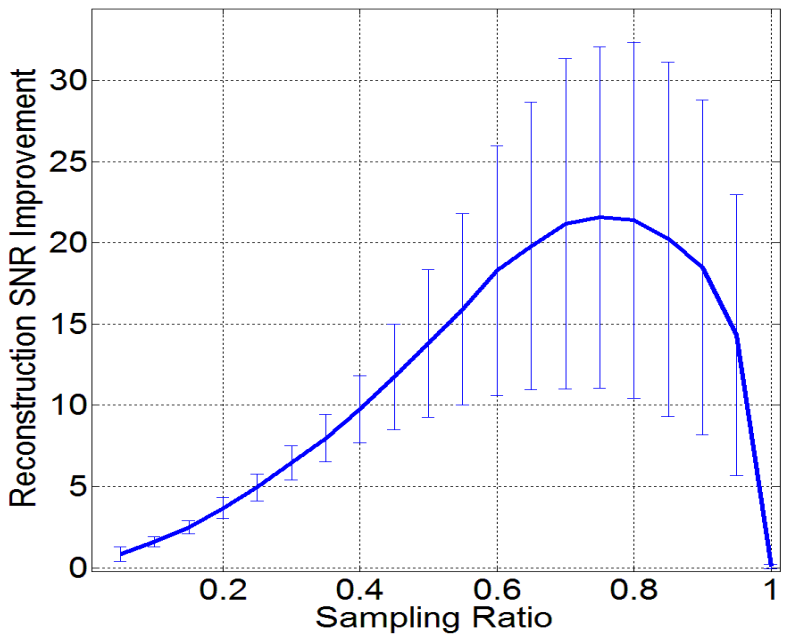

(b)

Fig. 6. Subplot (a) SNR of Ramp-Peak surface reconstruction as a function of sub-sampling ratio $r$. Here, the dashed and solid lines correspond to classic CS and DCS, respectively, and SNR=20 dB. Subplot (b) Reconstruction SNR improvement as a function of sub-sampling ratio across the dataset of Fig. 2. Here the curve depicts the average improvement of reconstruction results across the dataset and error bars denote the variation across the dataset.

at substantially lower noise levels. Finally, we note that a promising direction for future work on the subject would be to replace the use of wavelet analysis with more advanced (e.g., adaptively learned) sparse signal representations. Although the proposed algorithm can handle the above conditions, further experiments confirm algorithm quality degrades when deviated from suitable conditions for CS based approaches. Note the sampling operator is generally assumed to be a random sensing matrix in CS setting, while in practice only limited number of CS based approaches have been realized in hardware for CS encoding. Consequently, we either need a suitable encoding device or to improve the algorithm for the case of decimation downsampling to be able to use the proposed method. One direction for this purpose is to use a better sparsifying basis. It is pretty known that CS can work 


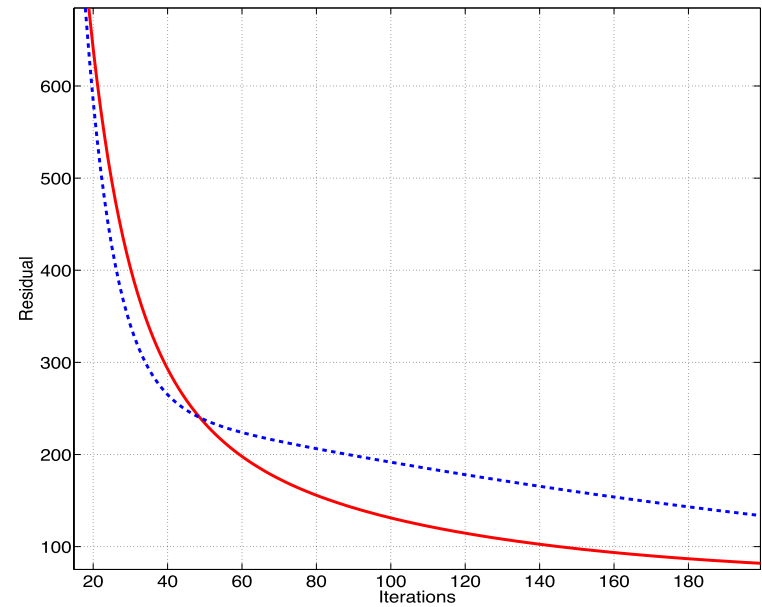

(a)

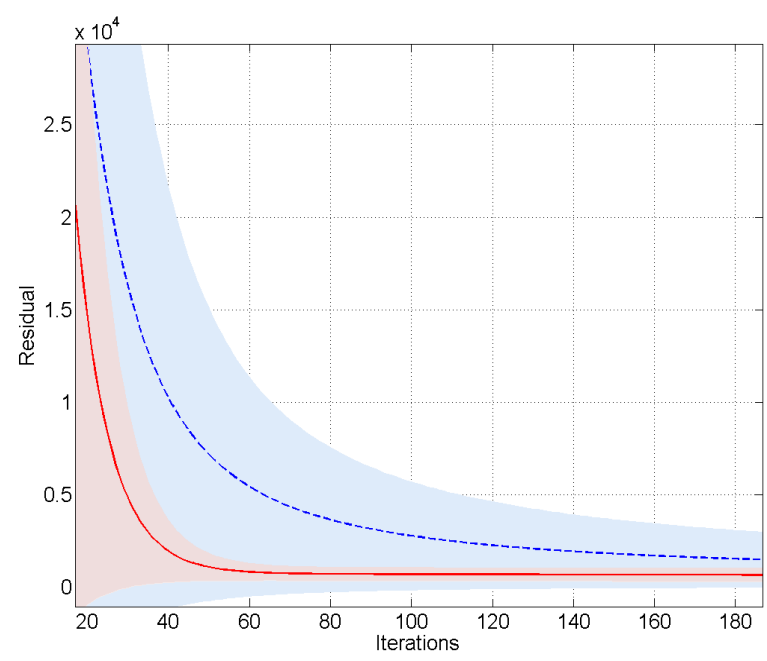

(b)

Fig. 7. Subplot (a) Convergence analysis of Ramp-Peak surface reconstruction obtained with different methods as a function of iterations. Here, the dashed and solid lines correspond to classic CS and DCS, respectively, $S N R=20$, and $r=0.5$. Subplot (b) Convergence analysis across the dataset of Fig. 2. Here the dashed and solid curves correspond to classic CS and DCS, respectively. The curves denote the average performance across dataset and the shaded area denotes the variation across the dataset.

better for the case of incoherent downsampling matrices. One can improve this quality by using a more suitable sparsifying basis. Another limitation of the proposed algorithm is noise model which is assumed to be Gaussian and cannot take into account outliers which exist in practical applications. One direction for future studies to improve the algorithm to become more suitable for the case of practical application.

\section{ACKNOWLEDGMENT}

The authors would like to acknowledge the anonymous reviewers for their valuable comments and suggestions which helped to improve the quality of the paper.

\section{REFERENCES}

[1] M. Rostami, O. Michailovich, and Z. Wang, "Gradient-based surface reconstruction using compressed sensing," in Proc. 19th IEEE Int. Conf. Image Process. (ICIP), Sep./Oct. 2012, pp. 913-916.
[2] A. Agrawal, R. Raskar, and R. Chellappa, "What is the range of surface reconstructions from a gradient field?" in Computer Vision. Berlin, Germany: Springer-Verlag, 2006, pp. 578-591.

[3] D. Reddy, A. Agrawal, and R. Chellappa, "Enforcing integrability by error correction using $\ell_{1}$-minimization," in Proc. IEEE Conf. Comput. Vis. Pattern Recognit., Jun. 2009, pp. 2350-2357.

[4] H. D. Tagare and R. I. P. deFigueiredo, "A theory of photometric stereo for a class of diffuse non-Lambertian surfaces," IEEE Trans. Pattern Anal. Mach. Intell., vol. 13, no. 2, pp. 133-152, Feb. 1991.

[5] F. Pernkopf and P. O'Leary, "Image acquisition techniques for automatic visual inspection of metallic surfaces," NDT \& E Int., vol. 36, no. 8, pp. 609-617, 2003.

[6] R. Zhang, P.-S. Tsai, J. E. Cryer, and M. Shah, "Shape-from-shading: A survey," IEEE Trans. Pattern Anal. Mach. Intell., vol. 21, no. 8, pp. 690-706, Aug. 1999.

[7] R. J. Woodham, "Photometric method for determining surface orientation from multiple images," in Shape From Shading, B. K. P. Horn, Ed. Cambridge, MA, USA: MIT Press, 1989, pp. 513-531.

[8] B. K. P. Horn and M. J. Brooks, "The variational approach to shape from shading," Comput. Vis., Graph., Image Process., vol. 33, no. 2, pp. 174-208, 1986.

[9] H. H. Bülthoff and H. A. Mallot, "Integration of depth modules: Stereo and shading," J. Opt. Soc. Amer. A, vol. 5, no. 10, pp. 1749-1758, 1988.

[10] R. T. Frankot and R. Chellappa, "A method for enforcing integrability in shape from shading algorithms," IEEE Trans. Pattern Anal. Mach. Intell., vol. 10, no. 4, pp. 439-451, Jul. 1988.

[11] N. Amenta, M. Bern, and M. Kamvysselis, "A new Voronoi-based surface reconstruction algorithm," in Proc. 25th Annu. Conf. Comput. Graph. Interact. Techn., 1998, pp. 415-421.

[12] J. Ma, "Compressed sensing for surface characterization and metrology," IEEE Trans. Instrum. Meas., vol. 59, no. 6, pp. 1600-1615, Jun. 2010.

[13] E. J. Candés, J. Romberg, and T. Tao, "Robust uncertainty principles: Exact signal reconstruction from highly incomplete frequency information," IEEE Trans. Inf. Theory, vol. 52, no. 2, pp. 489-509, Feb. 2006

[14] D. L. Donoho, "Compressed sensing," IEEE Trans. Inf. Theory, vol. 52, no. 4, pp. 1289-1306, Apr. 2006.

[15] P. Boufounos, "Compressive sensing for over-the-air ultrasound," in Proc. IEEE Int. Conf. Acoust., Speech, Signal Process. (ICASSP), May 2011, pp. 5972-5975.

[16] M. Hosseini and O. Michailovich, "Derivative compressive sampling with application to phase unwrapping," in Proc. EUSIPCO, Glasgow, U.K., Aug. 2009, pp. 115-119.

[17] M. Rostami, O. Michailovich, and Z. Wang, "Image deblurring using derivative compressed sensing for optical imaging application," IEEE Trans. Image Process., vol. 21, no. 7, pp. 3139-3149, Jul. 2012.

[18] M. Lustig, D. Donoho, and J. M. Pauly, "Sparse MRI: The application of compressed sensing for rapid MR imaging," Magn. Reson. Med., vol. 58, no. 6, pp. 1182-1195, 2007.

[19] D. Gross, Y.-K. Liu, S. T. Flammia, S. Becker, and J. Eisert, "Quantum state tomography via compressed sensing," Phys. Rev. Lett., vol. 105, no. 15 , p. 150401 , Oct. 2010.

[20] G.-H. Chen, J. Tang, and S. Leng, "Prior image constrained compressed sensing (PICCS): A method to accurately reconstruct dynamic CT images from highly undersampled projection data sets," Med. Phys., vol. 35, no. 2, pp. 660-663, 2008.

[21] Z. Du, A. Robles-Kelly, and F. Lu, "Robust surface reconstruction from gradient field using the L1 norm," in Proc. 9th Biennial Conf. Austral. Pattern Recognit. Soc. Digit. Image Comput. Techn. Appl., Dec. 2007, pp. 203-209.

[22] W. Xie, Y. Zhang, Charlie C. L. Wang, and R. C.-K. Chung, "Surfacefrom-gradients: An approach based on discrete geometry processin," in Proc. IEEE Comput. Soc. Conf. Comput. Vis. Pattern Recognit. (CVPR), Washington, DC, USA, Jun. 2014, pp. 2203-2210.

[23] H. Badri, H. Yahia, and D. Aboutajdine, "Robust surface reconstruction via triple sparsity," in Proc. IEEE Conf. CVPR, Jun. 2014, pp. 2291-2298.

[24] K. Kreutz-Delgado, J. F. Murray, B. D. Rao, K. Engan, T.-W. Lee, and T. J. Sejnowski, "Dictionary learning algorithms for sparse representation," Neural Comput., vol. 15, no. 2, pp. 349-396, 2003.

[25] A. Beck and M. Teboulle, "A fast iterative shrinkage-thresholding algorithm for linear inverse problems," SIAM J. Imag. Sci., vol. 2, no. 1, pp. 183-202, 2009.

[26] T. Blumensath and M. E. Davies, "Iterative hard thresholding for compressed sensing," Appl. Comput. Harmonic Anal., vol. 27, no. 3, pp. 265-274, 2009. 
[27] D. Needell and J. A. Tropp, "CoSaMP: Iterative signal recovery from incomplete and inaccurate samples," Appl. Comput. Harmonic Anal., vol. 26, no. 3, pp. 301-321, 2009.

[28] Y. Zhang, "Theory of compressive sensing via $\ell_{1}$-minimization: A non-RIP analysis and extensions," Dept. Comput. Appl. Math., Rice Univ., Houston, TX, USA, Tech. Rep. TR08-11, 2008, pp. 19-22.

[29] B. S. Kashin and V. N. Temlyakov, "A remark on compressed sensing," Math. Notes, vol. 82, nos. 5-6, pp. 748-755, 2007.

[30] S. A. Vavasis, "Derivation of compressive sensing theorems from the spherical section property," Dept. Combinatorics Optim., Univ. Waterloo, Waterloo, ON, Canada, Tech. Rep. CO769, 2009.

[31] A. d'Aspremont. (2011). "Sparse recovery, Kashin decomposition and conic programming." [Online]. Available: http://arxiv.org/abs/1101.3027

[32] T. T. Cai and A. Zhang, "Sharp RIP bound for sparse signal and low-rank matrix recovery," Appl. Comput. Harmonic Anal., vol. 35, pp. 74-93, Jan. 2013.

[33] L. Vandenberghe and S. Boyd, "Semidefinite programming," SIAM Rev., vol. 38, no. 1, pp. 49-95, 1996.

[34] M. Grant and S. Boyd. CVX: Matlab Software for Disciplined Convex Programming, Version 1.21. [Online]. Available: http://cvxr.com/cvx/, accessed 2011.

[35] D. Dayton, B. Pierson, B. Spielbusch, and J. Gonglewski, "Atmospheric structure function measurements with a Shack-Hartmann wave-front sensor," Opt. Lett., vol. 17, no. 24 pp. 1737-1739, 1992.

[36] M. R. Hestenes, "Multiplier and gradient methods," J. Optim. Theory Appl., vol. 4, no. 5, pp. 303-320, 1969.

[37] R. Tibshirani, "Regression shrinkage and selection via the lasso," J. Roy. Statist. Soc., Ser. B (Methodological), vol. 58, no. 1, pp. 267-288, 1996.

[38] E. N. Coleman, Jr., and R. Jain, "Obtaining 3-dimensional shape of textured and specular surfaces using four-source photometry," Comput. Graph. Image Process., vol. 18, no. 4, pp. 309-328, 1982.

[39] M. Kazhdan, M. Bolitho, and H. Hoppe, "Poisson surface reconstruction," in Proc. 4th Eurograph. Symp. Geometry Process., 2006, pp. 1-10.

[40] M. Kazhdan and H. Hoppe, "Streaming multigrid for gradient-domain operations on large images," ACM Trans. Graph., vol. 27, no. 3, 2008, Art. ID 21.

[41] M. Harker and P. O'Leary, "Least squares surface reconstruction from measured gradient fields," in Proc. IEEE Conf. Comput. Vis. Pattern Recognit. (CVPR), Jun. 2008, pp. 1-7.

[42] A. Agrawal, R. Chellappa, and R. Raskar, "An algebraic approach to surface reconstruction from gradient fields," in Proc. 10th IEEE Int. Conf. Comput. Vis. (ICCV), Oct. 2005, pp. 174-181.

[43] I. Daubechies, Ten Lectures on Wavelets (CBMS-NSF Regional Conference Series in Applied Mathematics). Philadelphia, PA, USA: SIAM, 1992.

[44] B. Karaçali and W. Snyder, "Noise reduction in surface reconstruction from a given gradient field," Int. J. Comput. Vis., vol. 60, no. 1, pp. 25-44, 2004.

[45] T. Bodenmüller and G. Hirzinger, "Online surface reconstruction from unorganized 3D-points for the DLR hand-guided scanner system," in Proc. 2nd Int. Symp. 3D Data Process., Vis., Transmiss. (3DPVT), Sep. 2004, pp. 285-292.

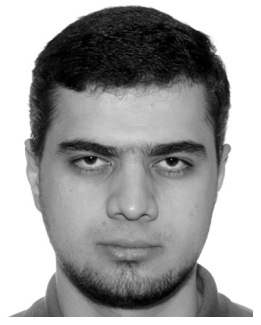

Mohammad Rostami (S'11) received the B.S. degrees in electrical engineering and pure mathematics from the Sharif University of Technology, Tehran, Iran, in 2010, and the M.Sc. degree in electrical and computer engineering from the University of Waterloo, Canada. $\mathrm{He}$ is currently pursuing the Ph.D. degree with the University of Pennsylvania, USA. His research interests include image and video processing/quality assessment, compressed sensing, computational neuroscience, and machine learning. He received the University of Waterloo Outstanding Achievement in Graduate Studies in Fall 2012 Convocation.

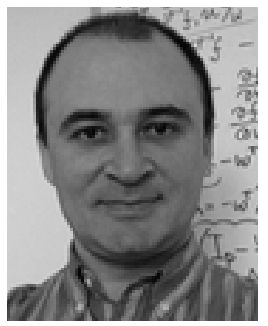

Oleg V. Michailovich (S'02-M'03) was born in Saratov, Russia, in 1972. He received the M.Sc. degree in electrical engineering from Saratov State University, Saratov, in 1994, and the M.Sc. and Ph.D. degrees in biomedical engineering from the Technion-Israel Institute of Technology, Haifa, Israel, in 2003. He is currently with the Department of Electrical and Computer Engineering, University of Waterloo, Waterloo, ON, Canada. His research interests include the application of image processing to various problems of image reconstruction, segmentation, inverse problems, nonparametric estimations, approximation theory, and multiresolution analysis.

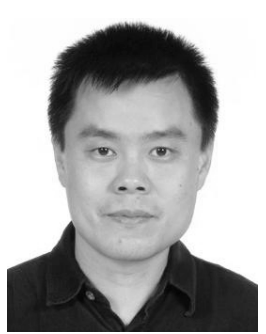

Zhou Wang (S'99-M'02-SM'12-F'14) received the Ph.D. degree in electrical and computer engineering from The University of Texas at Austin, in 2001. He is currently an Associate Professor with the Department of Electrical and Computer Engineering, University of Waterloo, Canada. He has over 100 publications in his research fields with over 23000 citations (the Google Scholar). His research interests include image processing, coding, and quality assessment, computational vision and pattern analysis, multimedia communications, and biomedical signal processing. $\mathrm{He}$ is a member of the IEEE Multimedia Signal Processing Technical Committee (2013-2015). He was a recipient of the 2014 NSERC E. W. R. Steacie Memorial Fellowship Award, the 2013 IEEE Signal Processing Best Magazine Paper Award, the 2009 IEEE Signal Processing Society Best Paper Award, the 2009 Ontario Early Researcher Award, and the ICIP 2008 IBM Best Student Paper Award (as a senior author). He served as an Associate Editor of the IEEE TRANSACTIONS on ImAge Processing (2009-2014) and the IEEE Signal Processing LETTERS (2006-2010), and a Guest Editor of the IEEE JOURNAL OF Selected Topics in Signal Processing (2013-2014 and 2007-2009), the EURASIP Journal of Image and Video Processing (2009-2010), and Signal, Image and Video Processing (2011-2013). He has been an Associate Editor of Pattern Recognition since 2006. 